\title{
MODELAGEM VOLUMÉTRICA DE ÁRVORES DE ACÁCIA-NEGRA EM POVOAMENTOS DE REGIÕES DO RIO GRANDE DO SUL
}

\section{VOLUMETRIC MODELING OF BLACK WATTLE STANDS LOCADED IN RIO GRANDE DO SUL REGIONS}

\author{
João Felipe Cardozo Martins, Jean Carlos Ribeiro ${ }^{1}$, César Luizon Padilha ${ }^{1}$, \\ Allan Libanio Pelissari ${ }^{1}$, Alexandre Behling ${ }^{1}$, Richardson Ribeiro ${ }^{2}$ \\ ${ }^{1}$ Universidade Federal do Paraná, Curitiba, Paraná, Brasil-jmartins.ufpr@gmail.com, \\ jeancarlosribeiro09@gmail.com, cesarluizonpadilha@gmail.com,allanpelissari@gmail.com \& \\ alexandre.behling@yahoo.com.br \\ ${ }^{2}$ Universidade Tecnológica Federal do Paraná, Pato Branco, Paraná, Brasil - prof.richard@gmail.com
}

\section{RESUMO}

A modelagem volumétrica para indivíduos florestais é recorrente na área florestal, havendo necessidade de reafirmar-se métodos e técnicas para mensurar volume e buscar melhores informações para as variáveis dendrométricas. A espécie acácia-negra (Acacia mearsii De Wild.) é reconhecida no sul do Brasil como uma espécie de alto valor comercial, apresentando a quarta maior área plantada de povoamentos florestais no Rio Grande do Sul., dessa forma, estudos a respeito da espécie são de grande importância para o setor florestal. 0 presente trabalho visou à obtenção de estimativas volumétricas individuais para árvores de acácia negra. Os modelos propostos por Hohenadl-Krenn. Kopesky-Gehrhardt, Spurr, Schumacher-Hall (não linear), Spurr (não linear), State (Australia) e Meyer foram ajustados. Foram empregados dados de árvores de acácia-negra com idades entre 1 e 10,75 anos, provenientes de um experimento nas regiões de Cristal, Encruzilhada do Sul e Piratini, estado do Rio Grande do Sul. Os dados foram divididos em 4 grupos distintos, de acordo com sua fase de crescimento (jovem, média-inicial, média-avançada e madura). Utilizou-se de 550 árvores para o ajuste de modelos, além de 30 indivíduos de cada grupo, previamente separados de maneira aleatória, para a validação do modelo de melhor desempenho. Os ajustes foram avaliados pelos seguintes critérios: erro padrão da estimativa em porcentagem $\left(S_{y x} \%\right)$, coeficientes de determinação múltiplo ajustados $\left(R^{2}{ }_{a j}\right)$ e gráficos de dispersão dos resíduos. Os modelos não lineares (Spurr e Schumacher-Hall) foram os que melhor se ajustaram aos dados em todas fases de crescimento, sendo o modelo proposto por Spurr não-linear para a classe Jovem e Schumacher-Hal não-linear para as demais classes de idades foram os que apresentaram os melhores desempenhos, com $\mathrm{S}_{\mathrm{yx}} \%$ variando de $2,87 \%$ a $4,48 \%$ e $\mathrm{R}^{2}{ }_{\text {aj }}$ entre 0,9742 e 0,9974 . Sua validação apresentou resultados satisfatórios para o erro padrão da estimativa e para o teste de Qui-quadrado, com valores de $S_{y x} \%$ variando entre 6,43\% e 7,57\%, enquanto $\chi^{2}$ variou de 0,0019 a 0,0126 . Sendo assim, observou-se que os modelos não lineares apresentaram resultados superiores aos obtidos com os modelos lineares.

PALAVRAS-CHAVE: Volume, Modelagem, Modelos não-lineares, Plantios florestais.

\section{ABSTRACT}

Volumetric modeling for forest individuals is recurrent in the forest area, requiring methods and techniques to measure volume and seek better information for dendrometric variables. The black wattle (Acacia mearsii De Wild.) Is recognized in southern Brazil as a species with high commercial value, presenting the fourth largest planted area of forest stands in Rio Grande do Sul. Thus, studies on the species are of great importance to the forestry sector. The present work aimed to obtain individual volumetric estimates for black wattle trees. The models proposed by Hohenadl-Krenn. Kopesky-Gehrhardt, Spurr, Schumacher-Hall (nonlinear), Spurr (nonlinear), State (Australia) and Meyer were adjusted. Data were used from Acacia mearsii De Wild. trees aged from 1 to 10.75 years, from an experiment in the regions of Cristal, Encruzilhada do Sul and Piratini, from state of Rio Grande do Sul. Data were divided into 4 distinct groups according to their growth phase (young, starting medium, advanced medium and mature). A total of 550 trees were used to adjust models, as well as 30 individuals from each group, previously randomly separated, to validate the best performance model. Adjustments were evaluated by the following criteria: standard error of percent estimation $\left(\mathrm{S}_{\mathrm{yx}} \%\right)$ adjusted multiple determination coefficients $\left(\mathrm{R}^{2}{ }_{\mathrm{aj}}\right)$ and residual scatter plots. The nonlinear models (Spurr and Schumacher-Hall) were the ones that best fit the data in all growth phases. The model proposed by Spurr nonlinear for Young Class and Schumacher-Hall nonlinear for other classes presented the best performances, with $\mathrm{S}_{\mathrm{yx}} \%$ ranging from $2.87 \%$ to $4.48 \%$ and $\mathrm{R}^{2}$ aj between 0.9742 and 0.9974 . Its validation presented satisfactory results for the estimation standard error and for the Chi-square test, with $\mathrm{S}_{\mathrm{yx}} \%$ values ranging from $6.43 \%$ to $7.57 \%$, while $\chi^{2}$ ranged from 0.0019 to 0.0126 . Thus, it was observed that the nonlinear models presented results superior to those obtained with the linear models.

KEYWORDS: Volume, Modeling, Nonlinear models, Forest plantations. 


\section{INTRODUÇÃO}

A compreensão do crescimento de florestas é importante não apenas para a obtenção dos estoques comerciais (THAINES et al., 2010) mas também possui alta relevância para predições da produção futura com base em poucas medições (BOTIN \& CARVALHO, 2015; CASTRO et al., 2016; SANQUETTA et al., 2016a). A predição do volume constitui numa das informações de maior importância para o diagnóstico do potencial de uma floresta. Diversos métodos foram desenvolvidos para estimar essa variável, entre eles o fator de forma, funções de afilamento e equações volumétricas, as quais, em sua maioria, são ajustadas a partir de dados obtidos para o diâmetro à altura do peito (d) e altura total (h) das árvores (MELLO et al., 2012).

Os modelos volumétricos consistem no método de uso mais recorrente na estimativa de volumes individuais ou até mesmo do estoque em florestais (ANDRADE et al., 2019). Há ainda a possibilidade de classificar os modelos volumétricos como: de (i) simples entrada, os quais possuem uma única variável independente, como o diâmetro; e as equações de (ii) dupla entrada, as quais estimam a variável dependente por meio de duas ou mais variáveis independentes, empregando o diâmetro e altura dos indivíduos, por exemplo (SANQUETTA et al., 2015b; SANQUETTA et al., 2016b).

Os modelos propostos por Hohenadl-Krenn, KopeskyGehrardt, Spurr, Schumacher-Hall (não linearizado), Spurr (não-linearizado); State (Austrália) e Meyer são bastante comuns na área florestal utilizados em diversos trabalhos como os de: Behling et al. (2018), Gonzáles et al. (2018), Lanzarin et al. (2018) e Martins et al., (2019). Segundo Andrade et al. (2019) dentre os 40 trabalhos, sobre modelos volumétricos, analisados em sua pesquisa, observou-se que os dez mais usuais são: Spurr (65\%), Schumacher-Hall logarítmico (55\%), Spurr logarítmico (45\%), Stoate e Kopezky-Gehrhardt (35\%), Naslung e Husch (30\%), Hohenadl-Krenn (27\%), Brenac (22,5\%) e Schumacher-Hall (20\%).

A espécie acácia-negra apresenta madeira com propriedades desejadas para produção de celulose, lenha e carvão para energia. A casca do fuste é utilizada para extração de taninos, ao passo que sua copa ganhou destaque em virtude da sua biomassa (VIEIRA; SCHUMACHER, 2010; MARTINS et al., 2019).

Estudos a respeito da espécie acácia-negra apresentam-se em certos trabalhos como: Schneider et al. (2000), Schneider; Tonini (2003), Sanquetta et al. (2015c), Sanquetta et al. (2016b) e Schikowski et al. (2018). Demonstrando a importância da espécie e de se abranger maiores conhecimentos sobre acácia-negra.

Nesse contexto, sendo que o ajuste de equações volumétricas auxilia na predição do volume de indivíduos de acácia-negra, objetiva-se com este trabalho:

a) Ajustar os modelos volumétricos de: i) HohenadlKrenn; ii) Kopeszky-Gehrhardt; iii) Spurr; iv) Schumacher-Hall (não linear); v) Spurr (não linear); vi) State (Austrália); e vii) Meyer;

b) Comparar os modelos volumétricos por meio das estatísticas: Coeficiente de determinação ajustado $\left(R^{2}{ }_{\text {aj. }}\right)$ e o Erro padrão da estimativa $\left(S_{y x}\right)$;

c) Avaliar os resíduos para o melhor modelo;

d) Avaliar os resultados estatísticos obtidos com os dados de validação.

A hipótese é que os modelos não lineares apresentam resultados superiores aos modelos lineares.

\section{MATERIAL E MÉTODOS}

\section{Origem dos dados}

A pesquisa foi conduzida em plantios de acácia-negra, estabelecidos no estado do Rio Grande do Sul, mais precisamente nas regiões dos municípios de Cristal, Encruzilhada do Sul e Piratini demonstradas na Figura 1 proposta por Martins et al. (2019). Segundo Alvares et al. (2016), as três cidades possuem classificação, segundo Köppen-Geiger, como sendo de clima quente $e$ temperado (Cfa).

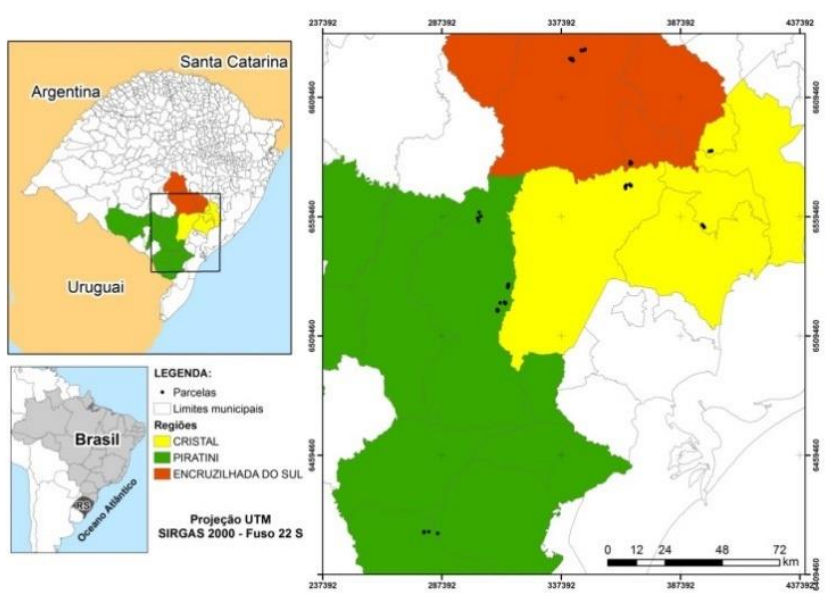

Figura 1. Áreas de cultivo de acácia-negra nas quais os povoamentos foram amostrados e a divisão dessa área em três regiões denominadas de Cristal, Encruzilhada do Sul e Piratini.

Os plantios apresentaram idades variando entre $1 \mathrm{e}$ 10,75 anos (Tabela 1). Foram amostrados 12 
povoamentos em sua totalidade, sendo que em cada região previamente mencionada se instalou quatro parcelas circulares com $400 \mathrm{~m}^{2}$. Dentro das parcelas houve a coleta das variáveis dendrométricas: volume (v), diâmetro à altura do peito (d) e a altura total (h) de 670 indivíduos. Dessa forma, 550 árvores foram usadas para o ajuste dos modelos volumétricas e os outros indivíduos para a validação dos melhores modelos.

Os dados de volume foram agrupados de acordo com fases de crescimento apresentados por Behling et al. (2018), conforme apresentado na Tabela 1.

Tabela 1. Idade dos povoamentos de Acácia-negra amostrados no estado do Rio Grande do Sul, Brasil.

\begin{tabular}{ccl}
\hline Local & Idade (anos) & \multicolumn{1}{c}{ Classe } \\
\hline \multirow{2}{*}{ Cristal } & 1,75 & Jovem \\
& 2,75 & Média Inicial \\
& 5,00 & Média Avançada \\
\hline \multirow{2}{*}{ Encruzilhada } & 10,08 & Madura \\
do Sul & 1,83 & Jovem \\
& 3,08 & Média Inicial \\
& 5,75 & Média Avançada \\
\hline \multirow{2}{*}{ Piratini } & 10,75 & Madura \\
& 1,00 & Jovem \\
& 2,33 & Média Inicial \\
& 5,25 & Média Avançada \\
& 9,83 & Madura \\
\hline
\end{tabular}

O volume alocado na árvore varia de acordo com a idade do povoamento, ou seja, tendências de indivíduos com dimensões e volumes maiores em idades superiores são comumente observadas (COLPINI et al., 2009; SANQUETTA et al., 2014; SANQUETTA et al., 2015a). Segundo Behling et al. (2018), a amplitude dos estratos não deve incluir tamanhos de amplitude maior que dois anos, visto que, dessa maneira, não seria evidenciados os efeitos das taxas de crescimento.

Modelos volumétricos

Sete modelos foram utilizados para estimar volume, sendo dois de simples entrada (1) e (2), em que a variável diâmetro à altura do peito (d) foi utilizada como variável dependente. Cinco modelos de dupla entrada (3 a 7) também foram testados, os quais, além da utilização de d, apresentam a variável dendrométrica altura total (h).

Modelos alométricos lineares e não lineares têm sido amplamente utilizados em estimativas na área florestal (CUNIA \& BRIGGS, 1984; REED \& GREEN, 1985; REED et al., 1996). As formulações matemáticas gerais para esses modelos são apresentadas na Tabela 2.
Tabela 2. Modelos volumétricos

\begin{tabular}{cc}
\hline Nome do autor & Modelo \\
\hline $\begin{array}{c}\text { Hohenadl-Krenn } \\
\text { Kopeszky- } \\
\text { Gehrhardt } \\
\text { Spurr }\end{array}$ & $v=\beta_{0}+\beta_{1} \cdot d+\beta_{2} \cdot d^{2}$ \\
$\begin{array}{c}\text { Schumacher-Hall } \\
\text { (não linear) }\end{array}$ & $v=\beta_{0}+\beta_{1} \cdot d^{2}$ \\
$\begin{array}{c}\text { Spurr (não linear) } \\
\text { State (Austrália) }\end{array}$ & $v=\beta_{0}+\beta_{1} \cdot d^{2} h$ \\
Meyer & $v=d^{\beta_{1}} \cdot h^{\beta_{2}}$ \\
\hline v é o volume $\left(\mathrm{m}^{3}\right) ;$ d é o diâmetro à altura do peito $(\mathrm{cm}) ; \mathrm{h}$ é a altura \\
total (m); $\beta_{0}, \beta_{1}, \beta_{2,}, \beta_{3}, \beta_{4}$ e $\beta_{5}$ são os parâmetros.
\end{tabular}

Nord-Larsen et al. (2017) afirmam que o uso de estimadores não lineares aumenta o viés dos modelos em comparação com estimativa por modelos lineares.

\section{Seleção da melhor equação}

A seleção do melhor modelo foi fundamentada nos critérios de seleção: (a) erro padrão da estimativa em porcentagem ( $\left.\mathrm{S}_{y x} \%\right),($ b) coeficiente de determinação ajustado $\left(R^{2}{ }^{2}\right)$, sendo que para os modelos não-lineares utilizou-se o pseudo $R^{2}$ consistindo no coeficiente de correlação elevado ao quadrado e (c) análise gráfica dos resíduos em $\mathrm{m}^{3}$.

\section{Validação dos modelos}

Para analisar se as equações volumétricas apresentadas são apropriadas para outros indivíduos não inclusos no processo de modelagem, utilizou-se 30 indivíduos da mesma classe de idade, optou-se por esse valor para padronizar e para manter uma quantidade de até $20 \%$ de cada classe, para que o número de indivíduos arbóreos seja representativo. Dessa forma, calculou-se o Qui-quadrado $\left(\chi^{2}\right)(1)$, com nível de significância $(\alpha)$ de 0,05 .

$$
\chi^{2}=\sum_{i=1}^{n} \frac{\left(y_{i}-\hat{y}_{i}\right)^{2}}{y_{i}}
$$

Em que: $y_{i}=$ volume real para cada observação; $\hat{y}_{\mathrm{i}}=$ volume estimado para cada observação.

Processamento dos dados

Todos os dados foram processados com auxílio dos softwares Microsoft EXCEL 2013 e SAS versão para estudantes.

\section{Procedimento de obtenção dos modelos não lineares}

Os modelos não lineares foram ajustados de modo diferenciado, utilizando-se o software estatístico SAS, no 
Algoritmo 1, a seguir, foi demonstrado o procedimento para implementar os modelos dentro do software.

Algoritmo 1. Implementação dos modelos não lineares de Schumacher-Hall e Spurr.

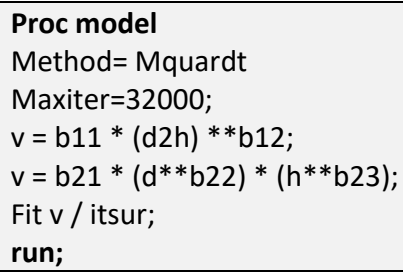

\section{RESULTADOS}

\section{Estatística descritiva}

As dimensões encontradas nas árvores apresentam forte relação sobre o volume. Sendo assim, a idade apresenta influência sobre as dimensões e consequentemente sobre o volume (MENSAH et al., 2016; WANG et al., 2018). Assim, optou-se por dividir os indivíduos em classes de idade (Tabela 1). As estatísticas descritivas foram destacadas na Tabela 3.

Tabela 3. Estatísticas descritivas das variáveis dendrométricas em povoamentos de acácia-negra no estado do Rio Grande do Sul, Brasil.

\begin{tabular}{|c|c|c|c|}
\hline Grupo & $v\left(m^{3}\right)$ & $d(\mathrm{~cm})$ & $h(m)$ \\
\hline \multicolumn{4}{|c|}{ Jovem } \\
\hline $\bar{x}$ & 0,0114 & 5,09 & 5,97 \\
\hline$S$ & 0,0108 & 2,76 & 2,57 \\
\hline $\mathrm{CV} \%$ & 94,46 & 54,18 & 43,11 \\
\hline IC superior & 0,0129 & 5,45 & 6,31 \\
\hline IC inferior & 0,0100 & 4,72 & 5,63 \\
\hline \multicolumn{4}{|c|}{ Média Inicial } \\
\hline $\bar{x}$ & 0,0463 & 9,27 & 11,91 \\
\hline$S$ & 0,0249 & 2,27 & 1,81 \\
\hline CV\% & 53,67 & 24,49 & 15,19 \\
\hline IC superior & 0,0499 & 9,60 & 12,17 \\
\hline IC inferior & 0,0428 & 8,95 & 11,65 \\
\hline \multicolumn{4}{|c|}{ Média Avançada } \\
\hline$\overline{\boldsymbol{x}}$ & 0,0899 & 11,39 & 15,13 \\
\hline$S$ & 0,0555 & 3,68 & 3,08 \\
\hline $\mathrm{CV} \%$ & 61,79 & 32,30 & 20,38 \\
\hline IC superior & 0,0982 & 11,94 & 15,59 \\
\hline IC inferior & 0,0816 & 10,84 & 14,67 \\
\hline \multicolumn{4}{|c|}{ Madura } \\
\hline$\overline{\bar{x}}$ & 0,1272 & 12,98 & 16,42 \\
\hline$S$ & 0,0847 & 4,03 & 2,98 \\
\hline CV\% & 66,61 & 31,08 & 18,12 \\
\hline IC superior & 0,1390 & 13,54 & 16,84 \\
\hline IC inferior & 0,1153 & 12,41 & 16,00 \\
\hline
\end{tabular}

\section{Ajuste dos modelos}

Os coeficientes de regressão estimados dos modelos ajustados são apresentados na Tabela 4.

Tabela 4. Modelos propostos por Hohenadl-Krenn, Kopeszky-Gehrhardt, Spurr, Schumacher-Hall (não linear), Spurr (não linear), State (Austrália) e Meyer para volume com casca de árvores de acácia-negra de povoamentos no Rio Grande do Sul, Brasil.

\begin{tabular}{|c|c|c|c|c|c|c|}
\hline Nome do autor & $\beta_{0}$ & $\beta_{1}$ & $\boldsymbol{\beta}_{2}$ & $\beta_{3}$ & $\boldsymbol{\beta}_{4}$ & $\beta_{5}$ \\
\hline \multicolumn{7}{|c|}{ Jovem } \\
\hline Hohenadl-Krenn & 0,0005 & $-0,0007$ & 0,0004 & & & \\
\hline Kopesky-Gehrhardt & $-0,00076$ & 0,00004 & & & & \\
\hline Spurr & 0,00247 & 0,0016 & & & & \\
\hline Spurr (não linear) & 0,00008 & 0,8903 & & & & \\
\hline $\begin{array}{l}\text { Schumacher-Hall } \\
\text { (não linear) }\end{array}$ & 0,00009 & 1,8231 & 0,8152 & & & \\
\hline State (Austrália) & $-0,00358$ & 0,0002 & 0,0011 & 0,0007 & & \\
\hline Meyer & 0,00303 & $-0,0011$ & 0,0001 & 0,0004 & 0,0003 & $-0,0009$ \\
\hline \multicolumn{7}{|c|}{ Média Inicial } \\
\hline Hohenadl-Krenn & $-0,0104$ & 0,0013 & 0,00049 & & & \\
\hline Kopesky-Gehrhardt & $-0,0041$ & 0,0006 & & & & \\
\hline Spurr & 0,0046 & $4 \mathrm{E}-05$ & & & & \\
\hline Spurr (não linear) & 0,0001 & 0,8937 & & & & \\
\hline $\begin{array}{l}\text { Schumacher-Hall } \\
\text { (não linear) }\end{array}$ & $9,3 \mathrm{E}-05$ & 1,8037 & 0,8538 & & & \\
\hline State (Austrália) & $-0,0081$ & 0,0002 & $2,2 \mathrm{E}-05$ & 0,00099 & & \\
\hline Meyer & 0,0102 & 0,0022 & $-0,0002$ & 0,0005 & 0,00002 & $-0,0038$ \\
\hline \multicolumn{7}{|c|}{ Média Avançada } \\
\hline Hohenadl-Krenn & 0,00652 & $-0,0032$ & 0,00083 & & & \\
\hline Kopesky-Gehrhardt & $-0,0041$ & 0,0006 & & & & \\
\hline Spurr & 0,00492 & $4 \mathrm{E}-05$ & & & & \\
\hline Spurr (não linear) & $6,5 \mathrm{E}-05$ & 0,9319 & & & & \\
\hline $\begin{array}{l}\text { Schumacher-Hall } \\
\text { (não linear) }\end{array}$ & 0,00006 & 1,8326 & 0,9923 & & & \\
\hline State (Austrália) & $-0,0065$ & $6 \mathrm{E}-05$ & 3,1E-05 & 0,0009 & & \\
\hline Meyer & $-0,0036$ & 0,0014 & $-6 \mathrm{E}-05$ & $6,9 \mathrm{E}-05$ & $3,3 \mathrm{E}-05$ & $-0,0002$ \\
\hline \multicolumn{7}{|c|}{ Madura } \\
\hline Hohenadl-Krenn & 0,00128 & $-0,0026$ & 0,0009 & & & \\
\hline Kopesky-Gehrhardt & $-0,0088$ & 0,0007 & & & & \\
\hline Spurr & 0,00582 & $4 \mathrm{E}-05$ & & & & \\
\hline Spurr (não linear) & $5,5 \mathrm{E}-05$ & 0,9569 & & & & \\
\hline $\begin{array}{l}\text { Schumacher-Hall } \\
\text { (não linear) }\end{array}$ & $4,5 \mathrm{E}-05$ & 1,8755 & 1,0589 & & & \\
\hline State (Austrália) & $-0,0048$ & $-5 E-05$ & $3,8 \mathrm{E}-05$ & 0,0009 & & \\
\hline Meyer & $-0,0303$ & 0,006 & $-0,0003$ & $-0,0003$ & $5,2 \mathrm{E}-05$ & 0,0020 \\
\hline
\end{tabular}


Os valores de $S_{y x} \%$ e $R^{2}$ aj dos modelos ajustados são apresentados na Tabela 5.

Tabela 5. Erro padrão das estimativas e o Coeficiente de Determinação calculados para os modelos propostos por Hohenadl-Krenn, KopeszkyGehrhardt, Spurr, Schumacher-Hall (não linear), Spurr (não linear), State (Austrália) e Meyer.

\begin{tabular}{|c|c|c|}
\hline Nome do autor & $\mathrm{S}_{\mathrm{yx}} \%$ & $\mathbf{R}^{\mathbf{2}}$ aj \\
\hline \multicolumn{3}{|c|}{ Jovem } \\
\hline Hohenadl-Krenn & 18,31 & 0,9624 \\
\hline Kopesky-Gehrhardt & 18,56 & 0,9614 \\
\hline Spurr & 15,74 & 0,9722 \\
\hline Spurr (não linear) & 3,90 & 0,9741 \\
\hline Schumacher-Hall (não linear) & 4,48 & 0,9742 \\
\hline State (Austrália) & 20,26 & 0,9540 \\
\hline Meyer & 13,93 & 0,9782 \\
\hline \multicolumn{3}{|c|}{ Média Inicial } \\
\hline Hohenadl-Krenn & 10,54 & 0,9615 \\
\hline Kopesky-Gehrhardt & 10,57 & 0,9612 \\
\hline Spurr & 8,50 & 0,9749 \\
\hline Spurr (não linear) & 2,81 & 0,9783 \\
\hline Schumacher-Hall (não linear) & 2,81 & 0,9784 \\
\hline State (Austrália) & 8,15 & 0,9769 \\
\hline Meyer & 7,60 & 0,9799 \\
\hline \multicolumn{3}{|c|}{ Média Avançada } \\
\hline Hohenadl-Krenn & 11,15 & 0,9674 \\
\hline Kopesky-Gehrhardt & 11,38 & 0,9661 \\
\hline Spurr & 6,31 & 0,9895 \\
\hline Spurr (não linear) & 2,47 & 0,9973 \\
\hline Schumacher-Hall (não linear) & 2,46 & 0,9974 \\
\hline State (Austrália) & 6,10 & 0,9902 \\
\hline Meyer & 6,11 & 0,9902 \\
\hline \multicolumn{3}{|c|}{ Madura } \\
\hline Hohenadl-Krenn & 11,41 & 0,9706 \\
\hline Kopesky-Gehrhardt & 11,47 & 0,9703 \\
\hline Spurr & 8,08 & 0,9853 \\
\hline Spurr (não linear) & 2,85 & 0,9927 \\
\hline Schumacher-Hall (não linear) & 2,87 & 0,9925 \\
\hline State (Austrália) & 4,31 & 0,9903 \\
\hline Meyer & 8,04 & 0,9854 \\
\hline
\end{tabular}

\section{Gráficos de resíduos}

A análise gráfica dos resíduos para o volume estimado dos modelos de Spurr (não linear) para a classe Jovem e Schumacher-Hall para as demais classes foram apresentadas na Figura 2.

\section{Validação da melhor equação}

Os modelos de Spurr (não linear) para classe Jovem e Schumacher-Hall para as demais classes, foram utilizados para validação, por serem os melhores modelos que se ajustaram para estimar o volume total com casca das árvores de acácia-negra utilizadas para o ajuste.
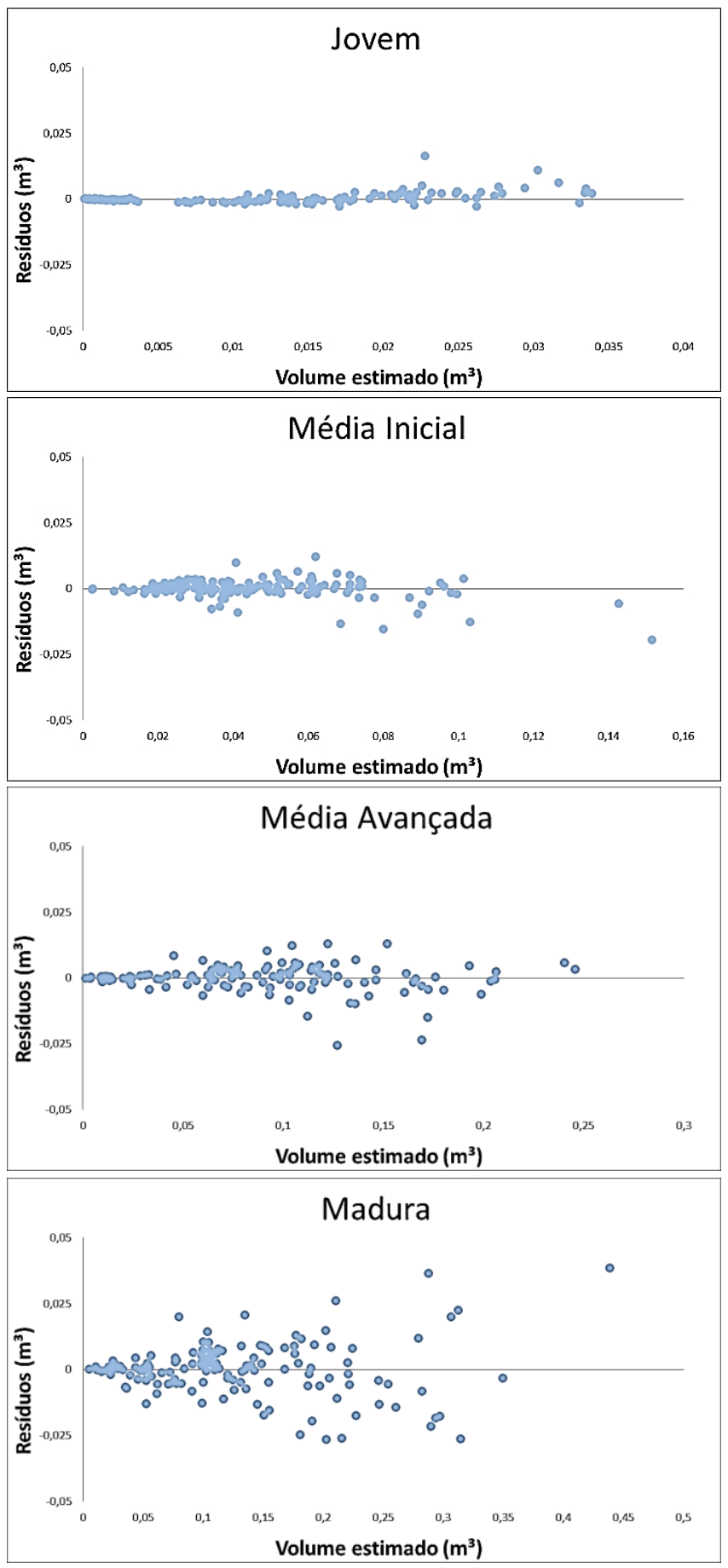

Figura 2. Distribuição dos resíduos de volume $\left(\mathrm{m}^{3}\right)$ em relação ao volume estimado para as classes em relação aos modelos de Schumacher-Hall.

Obteve-se os valores de Qui-quadrado $\left(\chi^{2}\right)$ calculado de $0,0019,0,0056,0,0080$ e 0,0126 para as equações que 
melhor se ajustaram aos dados dos grupos de idades Jovem, Média Inicial, Média Avançada e Madura, respectivamente. Os resultados não foram significativos para o $\chi^{2}$ tabelado $(17,71)$ com $95 \%$ de nível de significância, visto que foram inferiores ao tabelado. 0 erro padrão da estimativa ( $S_{y x} \%$ ) foi de 6,91\%, 7,57\%, $6,97 \%$ e $6,43 \%$, respectivamente.

\section{DISCUSSÃO}

Normalmente, as estimativas do número de árvores, área basal e volume de árvores são obtidas por unidade de área, a partir da idade (WOLFF II et al., 2019). Como forma de obter uma função genérica que estime volume para diferentes condições, têm-se empregado modelos que consideram outras variáveis dendrométricas, como a idade (LEITE et al., 2006; BEHLING et al., 2018; MARTINS et al., 2019). Os autores também descrevem diferenças significativas entre tratamentos com idades diferenciadas. Sendo assim, o emprego de classes de idades ao se modelar o volume de árvores de acácia-negra se demonstrou um procedimento assertivo.

Ao se analisar a Tabela 5e a Figura 2 observou-se que os modelos testados apresentaram ajustes aos dados superiores a 0,95. Houve uma variação bastante acentuada entre os modelos ajustados, tendo em vista que o $S_{y x} \%$ da estimativa variou entre $2,46 \%$ a $20,26 \%$, enquanto $\circ \mathrm{R}^{2}$ aj apresentou uma variação relativamente menor, de 0,9612 a 0,9982.

Foram modeladas duas funções de natureza não linear e a viabilidade no emprego de equações dessa natureza não se torna um fator excludente e acarreta na adição de aspectos positivos que não podem ser abordados com a regressão linear, contudo, cuidados devem ser tomados para confirmar a qualidade das funções (BEHLING et al., 2018).

De modo geral, as equações não lineares foram as que obtiveram os melhores resultados, dentre elas as equações de Schumacher-Hall apresentam o melhor comportamento residual, exceto para o caso da classe Jovem, nesse caso a equação de Spurr apresentou um melhor comportamento residual, pois estão bem distribuídos ao longo do eixo y e sem apresentar grandes desvios em torno do valor 0 , desse eixo, sendo o desejado quando trata-se de resíduos.

Resultados similares apresentados por Miguel et al. (2010), Pádua Chaves et al. (2011), Sales et al. (2015), Sanquetta et al. (2015a) e Sanquetta (2015b) descrevem que modelos não lineares apresentam melhores resultados que modelos lineares, o que corrobora e fundamenta os resultados apresentados nesse trabalho.
Ahrens (1982), Thomas et al. (2006), Barros \& Silva Júnior (2009) e Colpini et al. (2009) descrevem que vários modelos podem ser considerados ótimos para calcular volume de espécies florestais, sendo, que em muitos desses casos, Schumacher-Hall e Spurr estão presentes como os melhores modelos para estimar volume.

Parresol (1999) Sanquetta et al. (2014), Sanquetta et al. (2015a) e Behling et al. (2018) concluem que modelos não lineares resultaram em estimadores mais eficientes, reforçando os resultados apresentados nesta pesquisa. Isso ocorre visto que o método não considera as correlações contemporâneas, produzindo menor variância e parâmetro com erro padrão menor que outros tipos de análise (PARRESOL, 2001).

Sanquetta et al. (2016b) e Schikowski et al. (2018) demonstraram que modelos de Schumacher \& Hall apresentaram os melhores resultados para estimar volume de indivíduos de acácia-negra. Tais resultados corroboram com o apresentado no trabalho. Contudo vale a ressalva que ainda se tem poucos resultados sobre estimativas da variável volume ao se tratar da espécie acácia-negra, reforçando a importância desse e de outros trabalhos sobre essa finalidade.

As análises dos gráficos de resíduos para volume estimado das melhores equações (Figuras 2) corroboram com os resultados apresentados nos outros métodos estatísticos, demonstrando uma boa representação dos resíduos para as equações selecionadas.

Observou-se que, conforme o avanço da idade, o erro padrão da estimativa tende a ter uma redução nos seus ajustes. Tem-se um aumento nos resultados do coeficiente de determinação. Rolim et al. (2006) e Thomas et al. (2006) também apresentam que os resultados de simples entrada apresentaram os piores resultados.

A validação com dados independentes para o modelo de Spurr não linear e de Schumacher-Hall, confirmou a aplicabilidade das diferentes equações de volume estimadas para os plantios das regiões de Cristal, Encruzilhada do Sul e Piratini, no estado do Rio Grande do Sul. O Qui-quadrado calculado de 0,0019, 0,0056, 0,0080 e 0,0126, para o grupo de idade Jovem, Média Inicial, Média Avançada e Madura, respectivamente, foram considerados, não significativos (ns) para os valores apresentados na amostra de validação, confirmando que o modelo resultante também é considerado válido para estimar indivíduos, de mesma idade e desvio de diâmetro, não utilizados na modelagem da equação volumétrica.

\section{CONCLUSÕES}

Conforme esperado, as equações não lineares 
apresentaram as melhores estimativas para volume em todos os grupos de idades.

A comparação entre os modelos estimadores de volume demonstrou que as equações Spurr não linearizada para a classe Jovem e as de Schumacher \& Hall para as demais classes foram as que melhor se ajustaram aos dados de Acacia mearsii.

A validação de um conjunto independente refletiu a qualidade das predições e aumentou a credibilidade dos modelos, visto que o teste Qui-quadrado revelou que não houve diferença significativa nas estimativas das equações com os melhores resultados, demonstrando que é possível obter informações de volume com casca partindo de uma equação gerada com outros dados.

\section{AGRADECIMENTOS}

Os autores agradecem a empresa TANAC S.A./TANAGRO S.A. pela colaboração e fornecimento dos dados para elaboração do presente trabalho.

À Capes e ao CNPq pelo incentivo prestado à pesquisa por disponibilizar bolsas de pós-graduação.

\section{REFERÊNCIAS}

AHRENS, S. Importância da distribuição de resíduos de regressão na seleção de equações de volume. 40 Congresso Florestal Brasileiro, p.7-24, 1982.

ALVARES, C.A. et al. Köppen's climate classification map for Brazil. Meteorologische Zeitschrift, v.22, n.6, p.711-728, 2013.

ANDRADE, V.C.L. et al. Revisão sobre modelos volumétricos empregados em povoamentos florestais brasileiros. Advances in Forest Science, v.6, n.1, p.561-566, 2019.

BARROS, P.L.C.; SILVA JUNIOR, A.T. Equação de volume para árvores de uma floresta tropical densa no munícipio de Anapu, oeste do estado do Pará, Amaônia Oriental. Revista de Ciência Agrárias, v.51, n.1, p.115-12, 2009.

BEHLING, A. et al. Critical analyses when modeling tree biomass to ensure additivity of its componentes. Anais da Academia Brasileira de Ciências, v.90, n.2, p.1759-2690, 2018.

BOTIN, A.A.; CARVALHO, A.D. Reguladores de crescimento na produção de mudas florestais. Revista de Ciências Agroambientais, v.13, n.1, p.83-96, 2015.

CASTRO, R.V.O. et al. Modelagem do crescimento e da produção de povoamentos de eucalyptus em nível de distribuição da produção de povoamentos de eucalyptus em nível de distribuição diamétrica utilizando índice de local. Revista Árvore, v.40, n.1, p.107-116, 2016.

COLPINI, C. et al. Determinação do volume, do fator de forma e da porcentagem de casca de árvores individuais em uma Floresta Ombrófila Aberta na região noroeste de Mato Grosso. Acta Amazônica, v.39, n.1, p.97-104, 2009.
CUNIA, T. BRIGGS, R.D. Forcing additivity of biomass tables: some empirical results. Canadian Journal of Forest Research, v.14, p.376-384, 1984.

GONZÁLEZ, J.P.G. et al. Producción maderable de Hevea. Madera y Bosques, v.24, n.2, p.e2421867, 2018.

LANZARIN, K. et al. Crescimento e biomassa de indivíduos jovens de Cabrealea canjerana (Vell.) Mart. BIOFIX Scientific Journal, v.3, n.1, p.96-102, 2018.

LEITE, H.G. et al. Efeito do espaçamento e da idade sobre variáveis de povoamentos de Pinus taeda L. Revista Árvore, v.30, n.4, p.603-612, 2006

MARTINS, J.F.C. et al. Carbono nos components da biomassa de Acacia mearnsii De Wild. BIOFIX Scientific Journal, v.5, n.1, p.32$38,2019$.

MELLO, A.A. et al. Individual biomass and carbon equations for Mimosascabrella Benth. (bracatinga) in southern Brazil. Silva Fennica, v.46, n.3, p.333-343, 2012.

MENSAH, S. et al. Patterns of biomass allocation between foliage and woody structure: the effects of tree size and specific traits. Annals of Forest Research, v.59, n.49, p.49-60, 2016.

MIGUEL, E.P. et al. Ajuste de modelo volumétrico e desenvolvimento de fator de forma para plantios de Eucalyptus grandis localizados no municipal de Rio Verde-GO. Enciclopédia Biosfera, v.6, n.11, p.1-13, 2010.

NORD-LARSEN, Y. et al. Simultaneous estimation of biomass models for 13 tree species: effects of compartible additivity requirements. Canadian Journal of Forest Research, v.47, p.765776, 2017.

PARRESOL, B.R. Assessing tree and stand biomass: a review with examples and critical comparisons. Forest Science, v.45, p.573$593,1999$.

PARRESOL, B.R. Additivity of nonlinear biomass equations. Canadian Journal of Forest Research, v.31, p.865-878, 2001.

REED, D.D.; GREEN, E.J. A method of forcing additivity of biomass tables when using nonlinear models. Canadian Journal of Forest Research, v.15, p.1184-1187, 1985.

REED, D.D. et al. Above and belowground dry matter accumulation pattern derived fromdimensional biomass relationships. Forest Science, v.42, p.236-241, 1996.

ROLIM, S.G. et al. Modelos volumétricos para Floresta Nacional do Tapirapé-Aquirí, Serra dos Carajás (PA). Acta Amazonica, v.36, n.1, p.107-114, 2006.

SALES, F.D.C.V. et al. Ajuste de modelos volumétricos para o clone de Eucalyptus grandis $\times$ E. urophylla cultivados no Agrete de Pernanbuco. Floresta, v.45, n.4, p.663-670, 2015.

SANQUETTA, C.R. et al. Estoques de biomassa e carbono em povoamentos de acácia negra em diferentes idades no Rio Grande do Sul. Scientia Forestalis, v.42, n.103, p.361-370, 2014.

SANQUETTA, C.R. et al. Simultaneous estimation as alternative 
to independent modeling of tree biomass. Annals of Forest Science, v.72, p.1099-1112, 2015a.

SANQUETTA, C.R. et al. Modelling crown volume in Acacia mearnsii stands. African Journal of Agricultural Research, v.10, n.28, p.2756-2762, 2015c.

SANQUETTA, M.N.I. et al. Equações de biomassa para aroeirapimenteria (Schinus terebinthifolius RADDI). Enciclopédia Biosfera, v.11, n.22, p.832-840, 2015b.

SANQUETTA, C.R. et al. Modelos biológicos de crescimento em diâmetro para aroeira. Enciclopédia Biosfera, v.13, n.23, p.812$821,2016 a$.

SANQUETTA, M.N.I. et al. O uso de modelos alométricos tradicionais na estimative do peso seco aéreo individual para acacia negra. Enciclopédia Biosfera, v.13, n.23, p.842-854, 2016b.

SCHIKOWSKI, A.B. et al. Modeling of stem form and volume through machine learning. Anais da Academia Brasileira de Clências, v.90, n.4, p.3389-3401, 2018.

SCHNEIDER, P.R. et al. Crescimento da acácia-negra, Acacia mearnsii De Wild em diferentes espaçamentos. Ciência Florestal, v.10, n.2, p.101-112, 2000.

SCHNEIDER, P.R.; TONINI, H. Utilização de variáveis dummy em equações de volume para Acacia mearnsii De Wild. Ciência Florestal, v.13, n.2, p.121-129, 2003.

THAINES, F. et al. Equações para estimative de volume de madeira para a região da bacia do Rio Ituxi, Lábrea, AM. Pesquisa Florestal Brasileira, v.30, n.64, p.283-289, 2010.

THOMAS, C. et al. Comparação de equações volumétricas ajustadas com dados de cubagem e análise de tronco. Ciência Florestal, v.16, n.3, p.319-327, 2006.

VIEIRA, M.; SCHUMACHER, M.V. Deposição de serapilheira e de macronutrientes em um povoamento de acácia-negra (Acacia mearnsii De Wild.) no Rio Grande do Sul. Ciência Florestal, v.20, p.225-233, 2010.

WANG, X. et al. Additive tree biomass equation for Betula platyphylla Suk. Plantations in Northeast China. Annals of Forest Science, v.75, n.60, p.1-9, 2018.

WOLFF II, N.I. et al. Modelagem do crescimento e produção empregando ANATRO em Pinus taeda L. BIOFIX Scientific Journal, v.4, n.1, p.52-57, 2019.

Recebido em 05-11-2019 Aceito em 11-02-2020 